\title{
Autologous Conditioned Serum as a Novel Alternative Option in the Treatment of Unilateral Lumbar Radiculopathy: A Prospective Study
}

\author{
Ravi Kumar H. S. ${ }^{1}$, Vijay G. Goni ${ }^{2}$, Batra Y. K. ${ }^{3}$ \\ ${ }^{1}$ Department of Orthopaedics, Mandya Institute of Medical Sciences, Mandya, India \\ ${ }^{2}$ Department of Orthopaedics, Post Graduate Institute of Medical Education and Research, Chandigarh, India \\ ${ }^{3}$ Department of Anaesthesia, Post Graduate Institute of Medical Education and Research, Chandigarh, India
}

\begin{abstract}
Study Design: The study was conducted on patients who received autologous conditioned serum (ACS) as a line of treatment at the Orthopedics outpatient department of Post Graduate Institute of Medical Education and Research (PGIMER, Chandigarh) from January 2011 to June 2012. Of the 1,224 patients, 20 males or females were included in the study based on the inclusion and exclusion criteria. The institutional board of PGIMER approved the study before it was initiated.

Purpose: To study the efficacy of ACS in the treatment of unilateral lumbar radiculopathy.

Overview of Literature: Interleukin (IL)-1 appears to be of special importance among the cytokines identified in orthopedic diseases. ACS contains high concentrations of IL-1 receptor antagonist, antagonist to IL-1 in that is a biochemical 'sensitizer' of nerve roots in radiculopathy.

Methods: We included 20 patients with unilateral lumbar radiculopathy after obtaining informed consent. We prepared ACS as described by Meijer et al. Under bi-planar fluoroscopic imaging in anterior-posterior and lateral views, ACS was administered via epidural perineural technique. Patients in both groups were evaluated by quadruple visual analogue scale, straight leg raising test, revised Oswestry disability index, and 12-Item Short Form of Health Survey before and after epidural injections at 3 weeks, 3 months, and 6 months.

Results: There was a statistically significant change in all parameters from pre-injection to first, second, and third follow-up $(p<0.001)$. Conclusions: ACS can modify the disease course in addition to reducing pain, disability and improving general health.
\end{abstract}

Keywords: Autologous conditioned serum; Unilateral lumbar radiculopathy; Epidural perineural injection

\section{Introduction}

Low back pain (LBP) is the most common musculoskeletal disorder of industrialized society, and the most common cause of disability in persons younger than 45 years of age [1]. Disc degeneration is one of the main reasons for chronic LBP, accounting for 39\% incidence [2]. Disc herniation-associated radiculopathy is both a biochemical and a mechanical disorder. Nucleus pulposus contains a variety of inflammatory pain mediators, including phospholipase A2, nitric oxide and prostaglandin E [3].

Cytokines such as interleukin (IL)-1 have been identi-

Received Feb 15, 2015; Revised Mar 6, 2015; Accepted Mar 6, 2015

Corresponding author: Ravi Kumar H. S.

Department of Orthopaedics, Mandya Institute of Medical Sciences (MIMS),

KL-821, 20th cross v.v.nagar, Kallahalli, Mandya, Karnataka, 571401, India

Tel: +919035722667, Fax:+08232-231001, E-mail: drravikumarmmc1110@gmail.com 
fied as pivotal mediators of inflammatory and degenerative changes affecting components of the musculoskeletal system, including the lumbar spine. The presence of disc material in the epidural space initially results in direct toxic injury to the nerve root by chemical mediation, and subsequently, amplifies the intra- and extra-neural swelling that results in venous congestion and conduction block [4]. However, neither the size nor the location of the herniated disc correlates with pain [5].

Of the cytokines identified in orthopedic diseases, IL-1 appears to be of special importance [6]. The therapeutic use of IL-1 inhibitors in such diseases was proposed and has formed the basis for the development of new biological treatment modalities. Strategies for inhibiting the biological activities of IL-1 include the use of IL-1 receptor antagonist (Ra), soluble forms of IL-1 receptors, and type 1 cytokines such as IL-4, IL-10, and IL-13 that inhibit the synthesis of IL-1, increase the synthesis of IL-1Ra or both. The therapeutic use of cytokine inhibitors and growth factors was first proposed in the late 1970s and early 1980s. Growth factors, which target the revitalization of cytodamaged tissue like prolapsed disc function by modulating the underlying pathologies have an increasing role in the local treatment of lumbar radiculopathy [1].

Autologous conditioned serum (ACS) preparations are a source of anti-inflammatory cytokines, including IL-4, IL-10, IL-13 and IL-1Ra and also contain elevated concentrations of growth factors like fibroblast growth factor (FGF-2), hepatocyte growth factor and transforming growth factor (TGF- $\beta 1$ ) [6]. ACS contains high concentration of IL-1Ra, an antagonist to IL-1 that is a biochemical 'sensitizer' of nerve roots in radiculopathy [7]. Hence, ACS is a promising new treatment option for patients with unilateral lumbar radicular compression. The studies on efficacy of epidural perineural ACS in unilateral lumbar radiculopathy are limited. There are no such studies on the Indian population [4,7]. With this background, we designed a study to evaluate the efficacy of ACS in reducing pain and disability in unilateral lumbar radiculopathy.

\section{Materials and Methods}

The study was conducted on patients receiving ACS as a line of treatment at the Orthopedics outpatient department of Post Graduate Institute of Medical Education and Research (Chandigarh) from January 2011 to June 2012. We obtained ethical clearance from the institutional board before the study was initiated. Out of 1,224 patients, 110 male or female patients aged between 30 years to 60 years having moderate to severe low backache with radiation to unilateral lower limb of at least 6 weeks duration as determined by visual analogue scale (VAS) and positive straight leg raising test (SLRT) underwent clinical and radiological investigations. Of the 1,224 patients, 1,114 patients were excluded from the study based on history and clinical examination findings. The exclusion criteria included history of surgery on the lumbar spine and spinal canal stenosis, anticoagulant use and bleeding diathesis or altered coagulation, associated cervical myelopathy, systemic bone or joint illnesses, need of early surgery, epidural steroid injection to the affected nerve root in the prior 3 months, cortisone or opioid use in the prior 6 months, acute trauma, cauda equina syndrome, progressive neurological deficit, motor deficit, pain originating from an infection, allergies to corticosteroids or local anesthetics; and pregnancy and absence of substantial radicular pain as the presenting symptom.

Out of 110, 20 patients with radiological findings of abnormal disc degeneration as determined by X-ray based on Lane et al. [8] grading and magnetic resonance imaging (MRI) based on Pfirrmann et al. [9] grading were included in the study. Ninety patients were excluded based on the radiological findings like multiple disc level, bilateral involvement, canal stenosis, less grade in MRI and Xray, lack of a radiographically detectable abnormality. We included 20 patients with unilateral lumbar radiculopathy due to disc degeneration (lumbar spondylosis) after informed consent. The mean patient age was 37.15 years and the mean body mass index of the patient was $24.92 \mathrm{~kg} / \mathrm{m}^{2}$. We prepared ACS as described by Meijer et al. (Fig. 1) [10].

Under biplanar luoroscopic imaging in anterior-posterior and lateral views, ACS was administered through

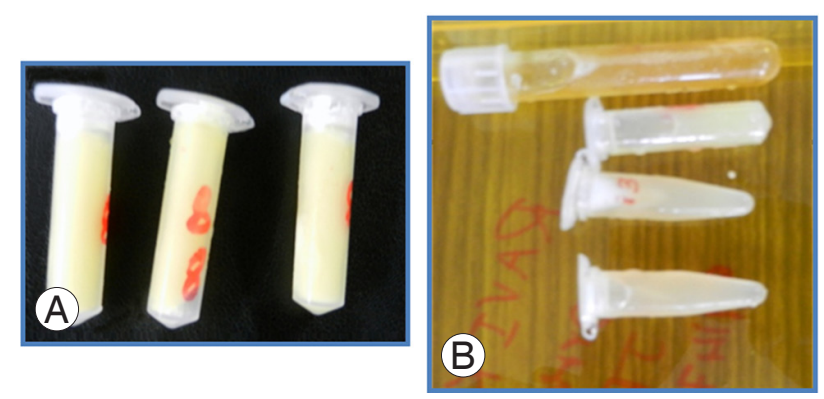

Fig. 1. Autologous conditioned serum (ACS). (A) ACS before thawing. (B) ACS after thawing. 
epidural perineural technique (Fig. 2). The entry site was marked with indelible ink. Sterile preparation was performed with alcohol followed by Betadine (povidoneiodine) solution. Local superficial anesthesia was by injection with $1 \%$ lidocaine buffered with a small amount of bicarbonate. The introducer needle for passing skin and interspinous ligaments was inserted $1 \mathrm{~cm}$ below and 2 $\mathrm{cm}$ contralateral, with an angle of $30^{\circ}-45^{\circ}$ to the midline. The 29-gauge needle then passed the ligamentum flavum and reached the lateral part of the anterior epidural space, which was recognized by bony contact. After locating the epidural space, under fluoroscopic guidance, contrast media Iohexol-180 (Omnipaque, GE Healthcare, Cork, Ireland), $2 \mathrm{~mL}$ was injected in the epidural space. The fluoroscopic images were obtained to confirm the spread. This step was immediately followed by $2 \mathrm{~mL}$ injection of ACS. Real-time fluoroscopy was used to verify that no medication would attain intravascular, subarachnoid, subdural, or intradiscal spread [11].

All the patients were kept under observation and the vitals were noted every 5 minutes for 30 minutes. The patient was advised to report in case of any adverse events. Patients were advised not to use nonsteroidal antiinflammatory drugs. Oral paracetamol $(500 \mathrm{mg}$, total dissolved solids) was used in case of discomfort. The patient was instructed to maintain a diary and to note when paracetamol was taken. The patient was advised to stop paracetamol (if consuming) 48 hours prior to followup assessment. A maximum of three injections at 7 days interval were given according to the clinical response. Twenty patients received an average of two injections, ranging from 1 to 3 injections.

Patients in both groups were evaluated by quadruple VAS subjective pain level or intensity of pain assessed on horizontal $10 \mathrm{~cm}$ scale was rated by the patients from 0 (no pain) to 10 (most pain possible), revised Oswestry disability index (RODI), and 12-Item Short Form of Health Survey (SF-12) to assess the level of restriction or inability of the patient to perform an activity in the manner or within range considered normal for a human as a consequence of disease. Patients were evaluated by the above mentioned scores after epidural injections at 3 weeks, 3 months, and 6 months.

Data was analyzed using computer statistical software IBM SPSS ver. 20.0 (IBM Co., Armonk, NY, USA). Data was presented as mean \pm standard deviation (SD) or median (range) where appropriate. Numerical variables were examined for normality. Intra-group comparisons were done using independent $t$-test and Student's $t$-test. Scores and skewed data were analyzed using Mann-Whitney's $U$-test. Incidence of complications was analyzed by chisquare test. A $p$-value of $<0.05$ was considered significant.

\section{Results}

The mean scores of all parameters and statistics at preinjection and follow-up were given in Table 1. Pairwise comparison of the parameters value at each time frame was shown in Table 2.

There was a statistically significant change in VAS from pre-injection to first, second, and third follow-up $(p<0.001)$. There was also a significant change from first
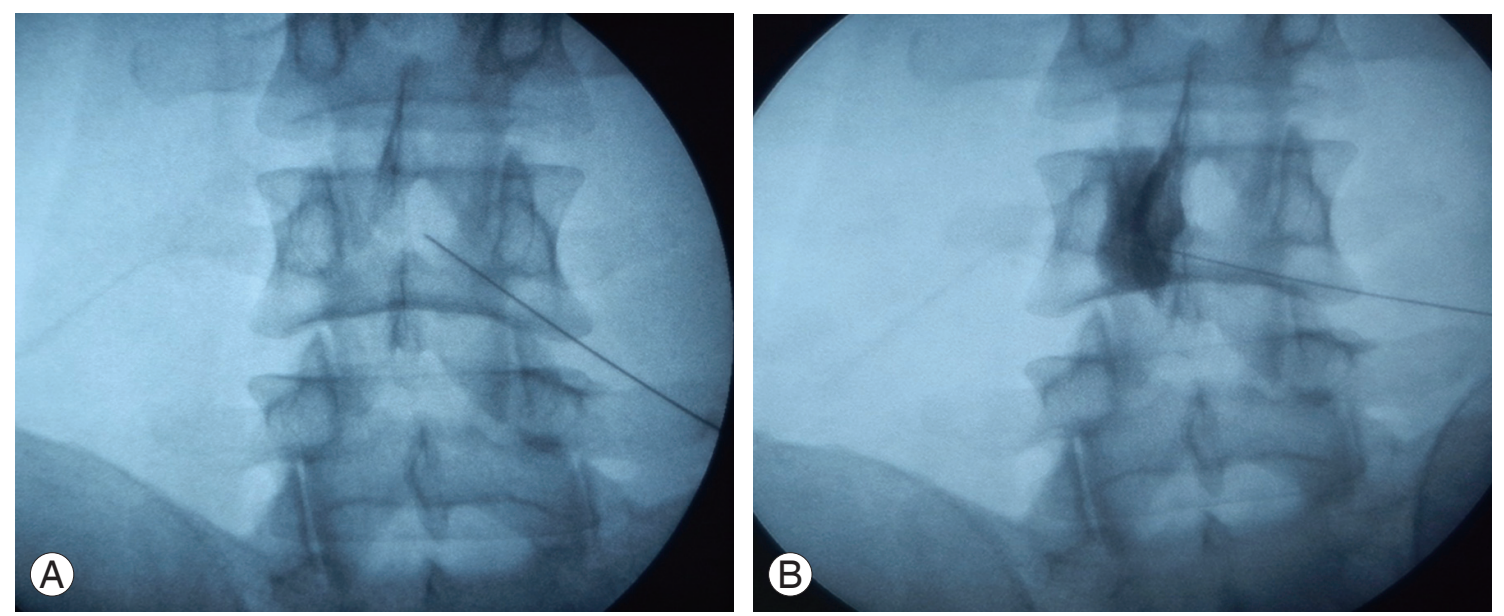

Fig. 2. Epidural perineural injection. 
Table 1. Mean values of parameters at different time frame

\begin{tabular}{lccccc} 
Time frame & VAS & SLRT & RODI & PCS & MCS \\
\hline Pre-injection & 6.95 & 42.00 & 27.90 & 27.25 & 36.59 \\
\hline First follow-up & 3.65 & 69.00 & 14.95 & 40.08 & 43.46 \\
\hline Second follow-up & 2.55 & 74.50 & 10.50 & 47.59 & 45.79 \\
Third follow-up & 2.00 & 76.00 & 8.50 & 49.32 & 47.51 \\
\hline
\end{tabular}

VAS, visual analogue scale; SLRT, straight leg raising test; RODI, revised Oswestry disability index; PCS, physical health component score; MCS, mental health component score.

Table 2. Mean difference and $p$-value of various parameters at different time frame

\begin{tabular}{|c|c|c|c|c|c|c|c|c|c|c|}
\hline \multirow[b]{2}{*}{ Time frame } & \multicolumn{2}{|c|}{ VAS } & \multicolumn{2}{|c|}{ SLRT } & \multicolumn{2}{|c|}{ RODI } & \multicolumn{2}{|c|}{ PCS } & \multicolumn{2}{|c|}{ MCS } \\
\hline & $\begin{array}{c}\text { Mean } \\
\text { difference }\end{array}$ & $p$-value & $\begin{array}{c}\text { Mean } \\
\text { difference }\end{array}$ & $p$-value & $\begin{array}{c}\text { Mean } \\
\text { difference }\end{array}$ & $p$-value & $\begin{array}{c}\text { Mean } \\
\text { difference }\end{array}$ & $p$-value & $\begin{array}{c}\text { Mean } \\
\text { difference }\end{array}$ & $p$-value \\
\hline \multicolumn{11}{|l|}{ Pre- injection } \\
\hline First follow-up & 3.30 & $<0.001$ & -29.23 & $<0.001$ & 12.95 & $<0.001$ & -12.83 & $<0.001$ & -6.89 & $<0.001$ \\
\hline Second follow-up & 4.40 & $<0.001$ & -31.03 & $<0.001$ & 17.40 & $<0.001$ & -20.34 & $<0.001$ & -9.20 & $<0.001$ \\
\hline Third follow-up & 4.95 & $<0.001$ & -28.46 & $<0.001$ & 19.40 & $<0.001$ & -22.07 & $<0.001$ & -10.9 & $<0.001$ \\
\hline \multicolumn{11}{|l|}{ First follow-up } \\
\hline Second follow-up & 1.10 & 0.001 & -1.80 & 0.399 & 4.45 & $<0.001$ & -7.51 & $<0.001$ & -2.32 & 0.039 \\
\hline Third follow-up & 1.65 & $<0.001$ & 0.77 & 0.772 & 6.45 & $<0.001$ & -9.24 & $<0.001$ & -4.03 & 0.001 \\
\hline \multicolumn{11}{|l|}{ Second follow-up } \\
\hline Third follow-up & 0.55 & 0.001 & 2.56 & 0.115 & 2.00 & 0.014 & -1.73 & 0.045 & -1.72 & 0.053 \\
\hline
\end{tabular}

VAS, visual analogue scale; SLRT, straight leg raising test; RODI, revised Oswestry disability index; PCS, physical health component score; MCS, mental health component score.

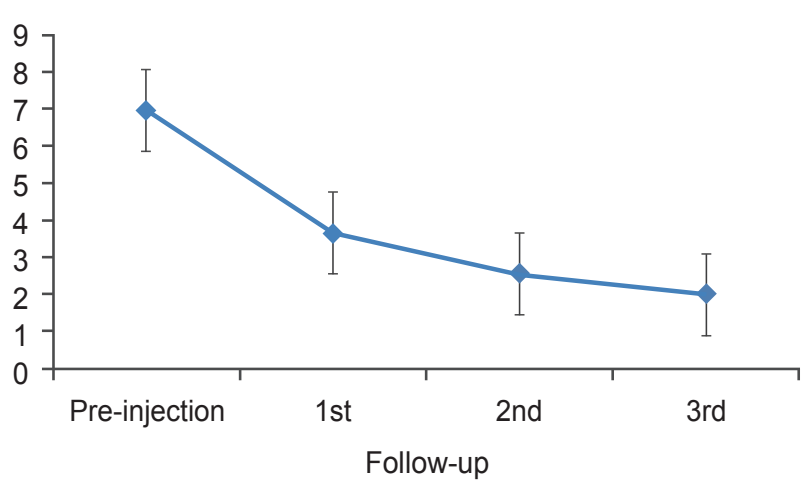

Fig. 3. Mean visual analogue scale scores at baseline and subsequent follow-ups.

follow-up to second follow-up ( $p=0.001)$ and from second follow-up to third follow-up ( $p=0.001$ ). The mean VAS decreased from pre-injection to first, second, and third follow-up. The trend was plotted in a graph (Fig. 3). There was a statistically significant change in SLRT scores from pre-injection to first, second, and third follow-up

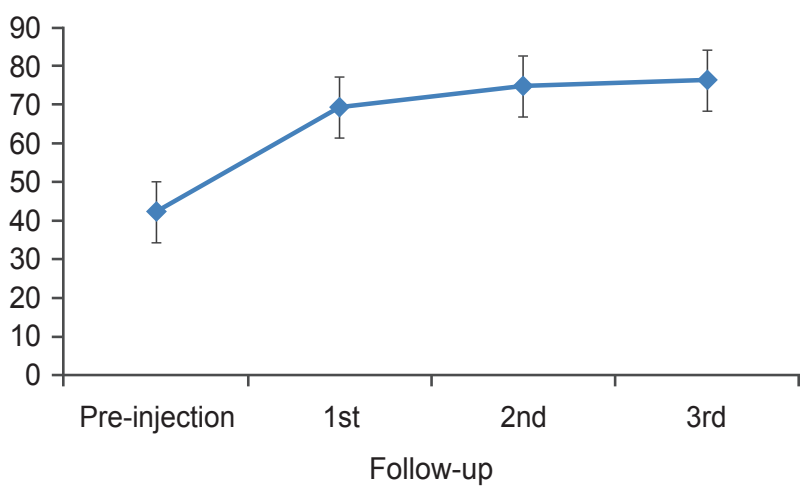

Fig. 4. Mean straight leg raising test scores at baseline and subsequent follow-ups.

$(p<0.001)$. There was no significant change from first follow-up to second and third follow-up ( $p=0.399$ and 0.772 , respectively) and from second follow-up to third followup $(p=0.115)$. The mean SLRT scores increased from preinjection to first, second, and third follow-up. The trend was plotted in a graph (Fig. 4). 
In our study, the RODI scores had a statistically significant change from pre-injection to first, second, and third follow-up $(p<0.001)$. There was also significant change from first follow-up to second follow-up $(p=0.001)$ and from second follow-up to third follow-up ( $p=0.001)$. The mean RODI scores decreased from pre injection to first, second, and third follow-up. The trend was shown in a graph (Fig. 5).

The SF-12 regarding general health has a total of 12 questions, with each question assigned a value ranging from 0 to 5 , with the total score ranging from 0 to 50 . Scoring for the physical health component score-12 (PCS12) and mental health component score-12 (MCS-12) of the SF-12 was performed using the SAS scoring program version 9.1.3 from the Medical Outcomes Trust, Massachusetts, USA. PCS-12 and MCS-12 range from 0 to 100 , with greater scores representing better health. Both the PCS-12 and MCS-12 are transformed into T-scores, normalized for the general United States population. The score for an individual or mean score for a group of patients is thus reported relative to a mean of 50.0 and a SD of 10.0 in the general United States population. Consequently, the SF-12 scores for the Indian-based population presented in this study are relative to the general United States population. The scores in the three follow-ups were compared with the pre-injection scores and the trend was studied in two groups to understand the effect of treatment on functional outcome [12].

PCS had a significant change from pre-injection to first, second, and third follow-up $(p<0.001)$. There was also significant change from first follow-up to second followup $(p=0.001)$ and from second follow-up to third followup $(p=0.001)$. The mean PCS increased from pre-injection to first, second, and third follow-ups. The trend was

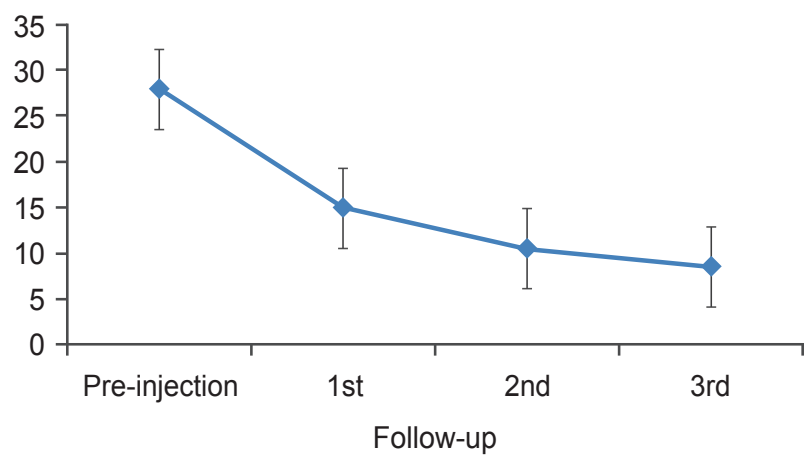

Fig. 5. Mean revised oswestry disability index scores at baseline and subsequent follow-ups. plotted in a graph (Fig. 6). MCS had a significant change from pre-injection to first, second, and third follow-ups $(p<0.001)$. There was no significant change from first follow-up to second follow-up ( $p=0.039$ ); and no significant change from second follow-up to third follow-up $(p=0.053)$. The mean MCS increased from pre-injection to first, second, and third follow-ups. The trend was plotted in graph (Fig. 7).

Five patients (20\%) had complications. Most of the complications were immediate and systemic rather than local and were of short duration lasting less than $30 \mathrm{~min}$ utes. The various complications noted were syncope, dizziness, headache, sweating, and tachycardia. None of the complications in either group were of severity or concern and subsided within half an hour when the patients were under observation. One patient had back stiffness after injection for 2 days that subsided spontaneously.

No serious complications such as infection, marked muscle atrophy, deep vein thrombosis, fever, hematoma, tissue hypertrophy, adhesion formation, or other major adverse events occurred among our study subjects. Since ACS

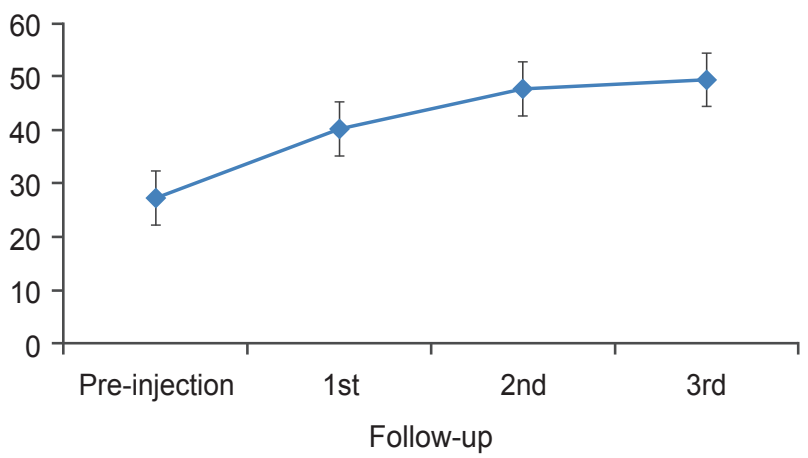

Fig. 6. Mean Physical Health Component Score at baseline and subsequent follow-ups.

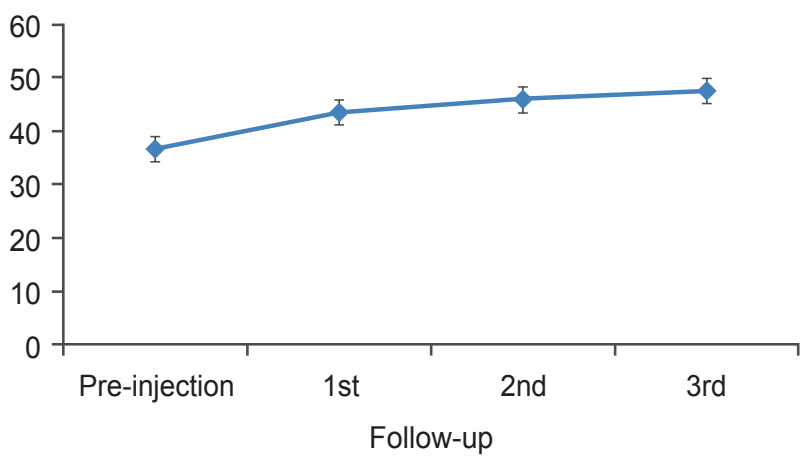

Fig. 7. Mean mental health component score at baseline and subsequent follow-ups. 
Table 3. Percent change in each parameter compared to baseline

\begin{tabular}{lccc} 
Follow-up & First (\%) & Second (\%) & Third (\%) \\
VAS & 47.48 & 63.31 & 71.22 \\
SLRT & -64.29 & -77.34 & -80.95 \\
RODI & 46.41 & 62.43 & 69.59 \\
PCS & -47.08 & -74.62 & -80.97 \\
MCS & -18.82 & -25.14 & -29.83 \\
\hline
\end{tabular}

VAS, visual analogue scale; SLRT, straight leg raising test; RODI, revised Oswestry disability index; PCS, physical health component score; MCS, mental health component score.

is an autologous preparation, the risk of introducing foreign material is effectively eliminated, although the entire procedure must be carried out in sterile conditions. The use of autologous blood products in this manner reduces the risk of transmissible infection and allergic reactions.

\section{Discussion}

The trends of all parameters were shown in Table 3. The primary outcome measure used in our study was VAS. Mean VAS scores decreased from pre-injection to further follow-ups. The results indicated a decrease in pain by $47.48 \%$ at first follow-up, by $63.31 \%$ at second follow-up and by $71.22 \%$ at third follow-up, as compared to baseline. All group comparisons were statistically significant $(p<0.001$ in all cases) in the framework of ordered testing (i.e., from time point of first follow-up to time point of third follow-up). The trend noted by Becker et al. [13] was also similar to our findings and showed a slight tendency of worsening in VAS in group with triamcinolone acetate and increase in VAS in the group with ACS scores from second month to sixth month, which was not significant.

There was an improvement in SLRT by $64.29 \%$ at first follow-up, by $77.34 \%$ at second follow-up and by $80.95 \%$ at third follow-up, as compared to baseline. All comparisons were statistically significant ( $p<0.001$ in all cases) in the framework of ordered testing (i.e., from time point of first follow-up to time point of third follow-up). Cocelli et al. [14] compared effectiveness of epidural triamcinolone and betamethasone with SLRT as a primary outcome measure. The pre-injection SLRT was 31.2 degrees and there was a significant improvement in first follow-up that was maintained at further follow-up.

RODI was the secondary outcome measure used in present study. Mean RODI scores showed a decreasing trend in all follow-up evaluation. There was an increased RODI percentage benefit by $46.41 \%$ at the first followup, by $62.43 \%$ at the second follow-up and by $69.59 \%$ at the third follow-up, as compared to baseline. Baseline functional capacity in a study by Rados et al. [15] was comparable for both the interlaminar and the transforaminal group ( $52 \%$ vs. $53 \%$, ODI converted into percent) when assessed using the ODI ( $p=0.647)$. At 6 months, both groups improved in function with an average of $39 \%$ for the interlaminar group and $38 \%$ for the transforaminal group, suggesting a change from severe to moderate disability scoring range. Cocelli et al. [14] compared the effectiveness of epidural triamcinolone and betamethasone. They reported that the ODI values were significantly decreased from the second week in both groups. Hence, in the present study, significant improvement of RODI might be attributed to the presence of increased amount of growth factors and use of fluoroscopic guided injection technique.

PCS-12 increased by $47.08 \%$ at first follow-up, by $74.62 \%$ at second follow-up and by $80.97 \%$ at third followup, as compared to baseline. MCS- 12 increased by $18.82 \%$ at first follow-up; by $25.14 \%$ at second follow-up, and by $29.83 \%$ at third follow-up, as compared to baseline. Birbara et al. [16] studied the efficacy of etoricoxib, a new cyclooxygenase-2 selective inhibitor in the treatment of chronic LBP, and used SF-12 as an outcome measure. Patients who were given etoricoxib did not experience a change from baseline that was significantly greater than placebo.

These studies collectively indicate that anti-inflammatory factors alone are not enough in improving the disability and general health in patients with LBP. Addition of growth factors enhances the biological environment and helps in attaining tissue homeostasis in patients with LBP. Thus, a cocktail of anti-inflammatory factors to- 
gether with growth factors play a vital role in improving the disability and general health. Hence, ACS can modify disease course in addition to reducing pain, disability and improving general health. Further investigation with larger sample size and longer follow-up is required to support the proposed hypothesis that might revolutionize the management of lumbar radiculopathy.

Limitations of this study were the lack of control group for comparing efficacy and short duration follow-up. This limitation can be overcome by proper design of future studies and the inclusion of post procedure MRI after 6 months to determine whether ACS has an effect on the disease course.

\section{Conclusions}

ACS can modify disease course in addition to reducing pain, disability and improving general health. Further studies are needed to confirm the results obtained and to understand the mechanism of the action. Further evaluations also required for long-term symptom improvement or the disease modifying properties of ACS in LBP.

\section{Conflict of Interest}

No potential conflict of interest relevant to this article was reported.

\section{References}

1. Mooney V, Saal JA, Saal JS. Evaluation and treatment of low back pain. Clin Symp 1996;48:1-32.

2. Anderson DG, Tannoury C. Molecular pathogenic factors in symptomatic disc degeneration. Spine J 2005;5(6 Suppl):260S-266S.

3. Spangfort EV. The lumbar disc herniation: a computer-aided analysis of 2,504 operations. Acta Orthop Scand Suppl 1972;142:1-95.

4. Saal JA. Natural history and nonoperative treatment of lumbar disc herniation. Spine (Phila Pa 1976) 1996;21(24 Suppl):2S-9S.

5. Saal JS. The role of inflammation in lumbar pain. Spine (Phila Pa 1976) 1995;20:1821-7.

6. Wright-Carpenter T, Klein P, Schaferhoff P, Appell HJ, Mir LM, Wehling P. Treatment of muscle injuries by local administration of autologous conditioned serum: a pilot study on sportsmen with muscle strains.
Int J Sports Med 2004;25:588-93.

7. Frisbie DD, Kawcak CE, Werpy NM, Park RD, McIlwraith CW. Clinical, biochemical, and histologic effects of intra-articular administration of autologous conditioned serum in horses with experimentally induced osteoarthritis. Am J Vet Res 2007;68:290-6.

8. Lane NE, Nevitt MC, Genant HK, Hochberg MC. Reliability of new indices of radiographic osteoarthritis of the hand and hip and lumbar disc degeneration. J Rheumatol 1993;20:1911-8.

9. Pfirrmann CW, Metzdorf A, Zanetti M, Hodler J, Boos N. Magnetic resonance classification of lumbar intervertebral disc degeneration. Spine (Phila Pa 1976) 2001;26:1873-8.

10. Meijer H, Reinecke J, Becker C, Tholen G, Wehling P. The production of anti-inflammatory cytokines in whole blood by physico-chemical induction. Inflamm Res 2003;52:404-7.

11. Kraemer J, Ludwig J, Bickert U, Owczarek V, Traupe M. Lumbar epidural perineural injection: a new technique. Eur Spine J 1997;6:357-61.

12. Ware J Jr, Kosinski M, Keller SD. A 12-Item ShortForm Health Survey: construction of scales and preliminary tests of reliability and validity. Med Care 1996;34:220-33.

13. Becker C, Heidersdorf S, Drewlo S, de Rodriguez SZ, Kramer J, Willburger RE. Efficacy of epidural perineural injections with autologous conditioned serum for lumbar radicular compression: an investigatorinitiated, prospective, double-blind, reference-controlled study. Spine (Phila Pa 1976) 2007;32:1803-8.

14. Cocelli LP, Karakurum G, Cebesoy O, Hakan Karadasli, Oner U. Clinical comparison of effectiveness of epidural triamcinolone and betamethasone in discal radiculalgia: a prospective, randomized study. J Musculoskelet Pain 2009;17:281-6.

15. Rados I, Sakic K, Fingler M, Kapural L. Efficacy of interlaminar vs transforaminal epidural steroid injection for the treatment of chronic unilateral radicular pain: prospective, randomized study. Pain Med 2011; 12:1316-21.

16. Birbara CA, Puopolo AD, Munoz DR, et al. Treatment of chronic low back pain with etoricoxib, a new cyclo-oxygenase-2 selective inhibitor: improvement in pain and disability: a randomized, placebocontrolled, 3-month trial. J Pain 2003;4:307-15. 